\title{
Formation, diffusion and accreting pollution of DB white dwarfs
}

\author{
Chunhua Zhu ${ }^{1,2}$, Helei Liu ${ }^{1,2}$, Zhaojun Wang ${ }^{1,2}$, and Guoliang L $\ddot{u ̈}^{1,2}$ \\ 1 School of Physical Science and Technology, Xinjiang University, Urumqi, 830046, China \\ e-mail: chunhuazhu@sina.cn \\ 2 Center for Theoretical Physics, Xinjiang University, Urumqi, 830046, China
}

July 27,2021

\begin{abstract}
Context. Over 1500 DBZ or DZ white dwarfs (WDs) have been observed so far, and polluted atmospheres with metal elements have been found among these WDs. The surface heavy element abundances of known DBZ or DZ WDs show an evolutionary sequence. The cooling, diffusion and accretion are important physical processes in the WD evolution which can alter the element abundances of the WD surface.

Aims. Using the stellar evolutionary code, we investigate the DB WD formation and the effects of input parameters-mixing length parameter $\left(\alpha_{\mathrm{MLT}}\right)$, thermohaline mixing efficiency $\left(\alpha_{\mathrm{th}}\right)$ and the metallicity $(Z)$-on the structures of these DB WDs. The impacts of convective zone mass $\left(M_{\mathrm{cvz}}\right)$, cooling timescales, diffusive timescales $\left(\tau_{\text {diff }}\right)$, and mass-accretion rate $\left(\dot{M}_{\mathrm{a}}\right)$ on the element abundances of the WDs' surfaces are discussed. By comparing the theoretical model results with observations, we try to understand the evolutionary sequence of the heavy element abundance on DBZ WD surfaces.

Methods. By using Modules for Experiments in Stellar Evolution, we create DB WDs, and simulate the element diffusion due to high gravitational fields and the metal-rich material accretion coming from the planet disrupted by the WD. Then, we calculate the element abundances of these DB WDs for the further comparison with observations.

Results. In our models, the input parameters $\left(\alpha_{\mathrm{MLT}}, \alpha_{\mathrm{th}}\right.$ and $\left.Z\right)$ have very weak effect on DB WD structures including interior temperatures, chemical profiles and convective zones. They hardly affect the evolution of the heavy elements on the surface of DB WDs. The mass-accretion rate and the effective temperature of DB WDs determine the abundances of heavy elements. The evolutionary sequence of Ca element for about 1500 observed DB or DBZ WDs cannot be explained by the model with a constant mass-accretion rate, but is consistent well with the model in which the mass-accretion rate decreases by one power law when $T_{\text {eff }}>10$ $\mathrm{kK}$ and slightly increases by another power law when $T_{\text {eff }}<10 \mathrm{kK}$.

Conclusions. The observed DB WD evolutionary sequence of heavy element abundances originates from WD cooling and the change of mass-accretion rate.
\end{abstract}

Key words. white dwarfs - stars: evolution - Accretion, accretion disks

\section{Introduction}

It is well known that single stars with initial mass between $\sim 1$ and $8 \mathrm{M}_{\odot}$ finally evolve into white dwarfs (WDs). Due to high gravitational fields ( $\log g \sim 8 \mathrm{~cm} \mathrm{~s}^{-2}$ ), the heavy elements on WDs' surfaces would diffuse downward during WD cooling. Usually, the timescale of diffusion $\left(\tau_{\text {diff }}\right)$ at the photosphere is about several to $10^{4}$ years Koester 2009), which is much shorter than cooling timescale $\left(t_{\text {cool }}\right)\left(\sim 10^{9} \mathrm{yr}\right)(\mathrm{e}$. g., Shapiro \& Teukolsky 1983; Zhu et al. 2019; Lü et al. 2017, 2020). Therefore, cool WDs should have pure hydrogen $(\mathrm{H})$ or helium (He) atmospheres. The former is called as DA WD, while the latter is called as DB WD. However, Zuckerman et al. (2003) pointed out that more than $25 \%$ of WDs are polluted by metal elements such as $\mathrm{Mg}, \mathrm{Fe}, \mathrm{Na}$. WDs are called as DAZ or DBZ WDs if their spectra show $\mathrm{H}$ or He lines with heavy element lines. When only heavy elements lines are displayed in the spectra, WDs are categorized as DZ type. Three different ways are proposed as three possible sources for the surface heavy elements of the WDs, such as primordial or fallback stellar material, interstellar medium, or debris disk produced by WD tidally disrupting rocky objects (i.e. planets)(e. g., Farihi 2016).

The pollution of WDs has been explained by ongoing accretion of planetary debris. A number of observa- tional evidences show the infrared emission from debris disk around polluted WDs(Jura 2003; Farihi et al. 2009; Girven et al. 2012; Vanderburg et al. 2015), thus these polluted WDs become unique laboratories for studying the interior composition of exoplanets(Zuckerman et al. 2007; Koester et al. 2014; Jura \& Young 2014). The chemical abundances detected on the surface of polluted WDs reflect the equilibrium between accretion for metal-rich material and diffusive sedimentation(Koester 2009; Bauer \& Bildsten 2018).

Dupuis et al. (1992) firstly explored the metal traces in WDs, and they also investigated the diffusion of metals accreted onto WDs(Dupuis et al. 1993a b). Koester \& Wilken (2006) calculated the diffusion timescales for some metals in DAZ WDs' atmosphere, and estimated the accretion rates for 38 DAZ WDs. Koester (2009) extended the above works to DAZ, DBZ and DZ WDs. Considering diffusion and thermohaline mixing, Wachlin et al. (2017) and Bauer \& Bildsten (2018) simulated the trace of metals for DAZ WDs. Bauer \& Bildsten (2019) discussed the effects of the mixing processes (including convection, gravitational sedimentation, overshoot, and thermohaline instability) on the diffusion. Using new WD envelope models and diffusion, Koester et al. (2020) investigated the atmospheres of carbon-rich WDs. In the theoretical models, the 
metal abundances of several polluted WDs can be explained well if suitable accretion rates are assumed(e. g., Koester 2009; Bauer \& Bildsten 2018). They also predicted that the metals would rapidly settle downward as soon as accretion stops. However, as shown in Koester (2009) and Bauer \& Bildsten (2018), the diffusion timescales of WDs increase with their effective temperature $\left(T_{\text {eff }}\right)$ decreasing. For a WD with $T_{\text {eff }}<10 \mathrm{kK}, \tau_{\text {dif }}$ is longer than about $10^{6} \mathrm{yr}$. Therefore it is imperfect to check long-timescale diffusion theory by only comparing theoretical results with several known cool WDs. A comparison involved a large observational sample of DB WDs with different $T_{\text {eff }}$ s becomes necessary.

Thanks to many large sky surveys, the number of observed WDs are dramatically increasing(e. g., Gaia Collaboration et al. 2016, 2018; Chambers et al. 2016; Blouin et al. 2019). Up to now, there are more than 60000 WDs in The Montreal White Dwarf Database(Dufour et al. 2017). One thousand and twenty three of them are DBZ or DZ WDs(Coutu et al. 2019). Observationally, Dufour et al. (2007) showed the spectroscopic and photometric data of $147 \mathrm{DZ}$ WDs with $T_{\text {eff }}$ between about $6 \mathrm{kK}$ and $12 \mathrm{kK}$. Based on SDSS DR10 and 12, Koester \& Kepler (2015) analyzed the data of 1107 DBZ WDs whose effective temperatures are between about $50 \mathrm{kK}$ and $11 \mathrm{kK}$. Hollands et al. (2017) identified 231 cool DZ WDs with $T_{\text {eff }}$ lower than $9 \mathrm{kK}$ in SDSS DR12. They discussed the distribution of $\log [\mathrm{Ca} / \mathrm{He}]$ vs. $T_{\text {eff }}$ for the three samples (See Figure 11 of Hollands et al. (2017)). At about $T_{\text {eff }}>10 \mathrm{kK}, \mathrm{Ca}$ abundances rapidly decrease with $T_{\text {eff }}$ declining. Koester \& Kepler (2015) suggested that this trend should be relative to the mass-accretion rates. However, $\mathrm{Ca}$ abundances of DZ WDs with $T_{\text {eff }}$ between $10 \mathrm{kK}$ and $8 \mathrm{kK}$ increase by about 100 times. Hollands et al. (2017) considered that this sharp increase might result from the decrease of convective zone mass $\left(M_{\mathrm{cvz}}\right)$ or the increase of $\tau_{\text {diff. The second downwards }}$ trend of Ca abundance with $T_{\text {eff }}$ appears between $9 \mathrm{kK}$ and $4 \mathrm{kK}$. Hollands et al. (2018) suggested that the trend is relative to $M_{\mathrm{cvz}}$ or $\tau_{\text {diff }}$.

Compared with DAZ WDs whose metal pollution is monotonically decreasing with $T_{\text {eff }}$ declining(Koester et al. 2014), DBZ WDs have more complicated progresses for metal pollution. The main reason is that DB WDs undergo different formation channels, and have distinctive interior structures. In this work, employing the stellar evolution code, we investigate the physical mechanisms to explain the surface metal abundance of the polluted DB WD. The model descriptions are given in section 2. DB WD's properties and their accretion pollution are shown and discussed in sections 3 and 4 . The paper is closed with conclusions in section 5 .

\section{Models}

In the present paper, we use Modules for Experiments in Stellar Evolution (MESA, [rev. 12115]; Paxton et al. (2011, 2013, 2015, 2018, 2019)) to create He-rich WDs without $\mathrm{H}$ which are noted as DB WDs, simulate the element diffusion within them and metal-rich material accretion. There are many factors to change the element abundances on the WD surface. Bauer \& Bildsten (2019) discussed the effects of convection, thermohaline instability, gravitational diffusion and rotation on the element mixing of WDs. Because the rotations velocities of most isolated WDs observationally are low (Berger et al. 2005; Kawaler 2015; Hermes et al. 2017), we do not consider the rotation. Convection directly determines the timescale of element diffusion Koester 2009; Bauer \& Bildsten 2018). In the present paper, we adopt the ML2 convection
Table 1. All cases in the present paper are simulated. The first column gives the case number. Columns 2, 3 and 4 show the values of input parameters $\alpha_{\mathrm{MLT}}, \alpha_{\mathrm{th}}$ and $Z$, respectively.

\begin{tabular}{cccc}
\hline Cases & $\alpha_{\text {MLT }}$ & $\alpha_{\text {th }}$ & $Z$ \\
case 1 & 1.8 & 1 & 0.02 \\
case 2 & 0.8 & 1 & 0.02 \\
case 3 & 1.8 & 0 & 0.02 \\
case 4 & 1.8 & 1000 & 0.02 \\
case 5 & 0.8 & 1 & 0.001 \\
\hline
\end{tabular}

prescription(Bohm \& Cassinelli 1971; Tassoul et al. 1990), and use Ledoux criterion for convection. The table named as 'DB_WD_tau_25' in MESA ( It is helium dominated atmosphere table for DB WDs) is used to calculate the DB WD atmosphere boundary. The size of convective zone depends on mixing length parameter ( $\left.\alpha_{\mathrm{MLT}}\right)$. In order to discuss its effect, we take $\alpha_{\text {MLT }}$ as 0.8 and 1.8 in different simulations, respectively.

Deal et al. (2013) and Wachlin et al. (2017) considered that thermohaline mixing can change the element abundances on the surfaces of polluted WDs. MESA adopts the method of Kippenhahn et al. (1980) to calculate the effects of thermohaline mixing, in which parameter $\alpha_{\text {th }}$ is used to give mixing efficiency. In our work, $\alpha_{\text {th }}$ is taken as 0,1 and 1000 in different calculations for testing its effects.

Schatzman (1945) suggested that the high gravitational fields in cool WDs should result in the downward diffusion of heavy elements. By resolving the Burgers equations which give multicomponent fluid's evolutions(Burgers 1969), Thoul et al. (1994) investigated the element diffusion in the interior of the Sun. Using the approach in Thoul et al. (1994), MESA can calculate the chemical diffusion in stellar interior(Paxton et al. 2015, 2018). The diffusion coefficients originated from Paquette et al. (1986) and updated by Stanton \& Murillo (2016) are used in our models.

Similarly, metallicity $(Z)$ can also affect stellar evolutions and WD properties. Here, Table 1 gives all cases in which different input parameters are considered.

\section{Formation and structures of DB WDs}

Many observations have showed that there are some $\mathrm{H}$ elements in the atmospheres of DBZ or DZ WDs (Voss et al. 2007; Koester \& Kepler 2015; Coutu et al. 2019). However, the ratios of $\mathrm{H}$ to $\mathrm{He}$ abundance estimated by these observations are lower than about $10^{-2}$. These $\mathrm{H}$ elements maybe continuously be accreted by DBZ or DZ WDs from interstellar medium (Voss et al. 2007; Koester \& Kepler 2015). Therefore, there may be no $\mathrm{H}$ elements left in the atmospheres of DB WDs when they form.

Usually, the range of DBZ or DZ WDs' masses is between about 0.4 and $1.0 \mathrm{M}_{\odot}$ and their mass distribution has a peak around 0.6 M ${ }_{\odot}$ (e. g., Han 1998; Han et al. 2000; Coutu et al. 2019). Take 0.6 M $\odot$ DB WD created by main sequence (MS) star under input parameters in case 1 as an example, we give all details for creating DB WDs by the following steps:

(i)The first step is showed by the black line in the left-top panel of Figure 1] The $3.5 \mathrm{M}_{\odot}$ MS star begins to normally evolve, that is, $\mathrm{H}$ starts to burn in the stellar core. The massloss rate $(\dot{M})$ is calculated by 'Dutch' scheme(Paxton et al. 2011), in which $\dot{M}$ of hot and cool stars is given by Nieuwenhuijzen \& de Jager (1990); Nugis \& Lamers (2000); Vink et al. (2001); Glebbeek et al. (2009) and Reimers (1975), 

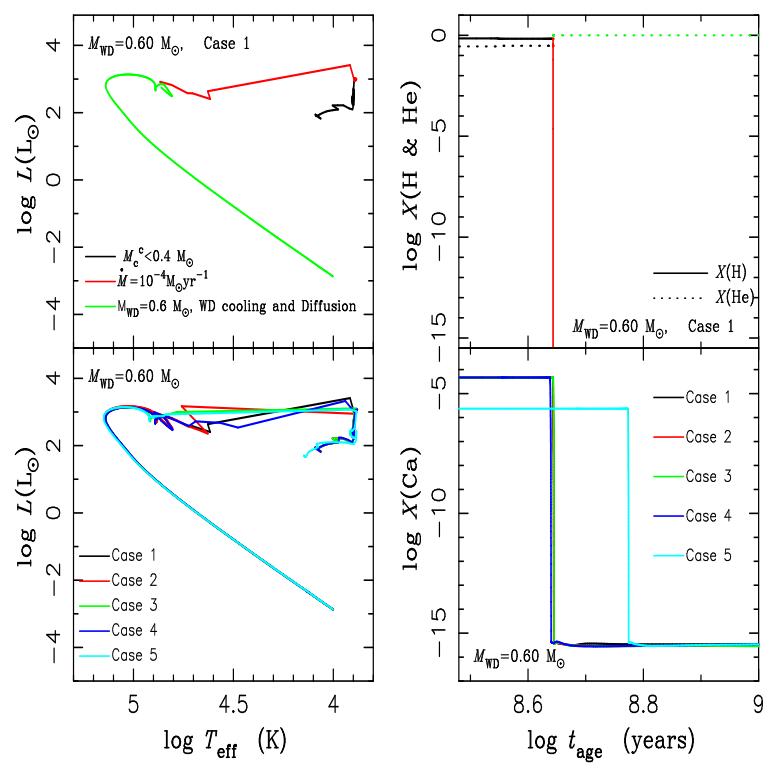

Fig. 1. The 0.6 $\mathrm{M}_{\odot} \mathrm{DB}$ WD produced by main sequence star with an initial mass of $3.5 \mathrm{M}_{\odot}$. The left-top panel gives the evolution in $\mathrm{HR}$ diagram for the star in case $1\left(\alpha_{\mathrm{MLT}}=1.8, \alpha_{\mathrm{th}}=1\right.$ and $\left.Z=0.02\right)$, in which the lines with different colors represent different evolutionary phases. The left-bottom panel shows the evolutions in HR diagram for stars with different input parameters which are given by different colors. The right-top panel is similar with the left-top panel, but for evolution of $\mathrm{H}$ and $\mathrm{He}$ abundances on the stellar surface. The right-bottom panel is similar with the left-bottom panel, but for evolution of $\mathrm{Ca}$ abundance on the stellar surfaces. The details can be seen in the text.

respectively. The element mixing is mainly determined by convection and thermohaline instability. At this phase, in order to save CPU time, we do not consider gravitational diffusion.

(ii)The second step is showed by the red line in the left-top panel of Figure 1. We artificially enhance the mass-loss rate up to $10^{-4} \mathrm{M}_{\odot} \mathrm{yr}^{-1}$ when He-core mass is larger than $0.6 \mathrm{M}_{\odot}$. The $\mathrm{H}$-rich envelope is rapidly stripped, and the star evolves into $\mathrm{He}$ star. As the red lines in the right-top panel of Figure 1 shows, the $\mathrm{H}$ abundance $(X(\mathrm{H}))$ on the stellar surface decreases from about 0.7 to about lower than $10^{-15}$, while $X(\mathrm{He})$ increases up to about 0.98 .

(iii)The third step is WD cooling, which is given by the green line. At this time, all $\mathrm{H}$ element almost is lost. He element is lightest, and it floats upward stellar surface by gravitational settling. A DB WD is created.

The left-bottom panel of Figure 1 shows the evolution in HR diagram for the star with different $\alpha_{\mathrm{MLT}}, \alpha_{\mathrm{th}}$ and $Z$. Obviously, the effects of input parameters on evolutionary tracks are negligible. The right-bottom panel gives the change of $X(\mathrm{Ca})$ on the stellar surface. $X(\mathrm{Ca})$ on the stellar surface starts to reduce because of gravitational sedimentation at WD cooling phase.

Using similar method, we also create DB WDs with 0.4 and $0.8 \mathrm{M}_{\odot}$, which are showed in Figure 2. The changes of $X(\mathrm{H})$, $X(\mathrm{He})$ and $X(\mathrm{Ca})$ on these WD surfaces are given, too.

As the left-bottom panel of Figure 1 shows, the cooling tracks of DB WDs are hardly affected by the input parameters. Similarly, the effects of these input parameters on DB WD internal structures can be negligible.

In Figure 3, we find that the profiles of the temperature, convective velocity $\left(v_{\text {cov }}\right)$, He abundance $(X(\mathrm{He}))$ and the abundance ratio of $\mathrm{Ca}$ to $\mathrm{He}([\mathrm{Ca} / \mathrm{He}])$ for $0.6 \mathrm{M}_{\odot} \mathrm{DB} W D$ at the same effective temperature are similar. Due to the strong gravitational diffusion of WD, heavy elements sink down and light He ele-
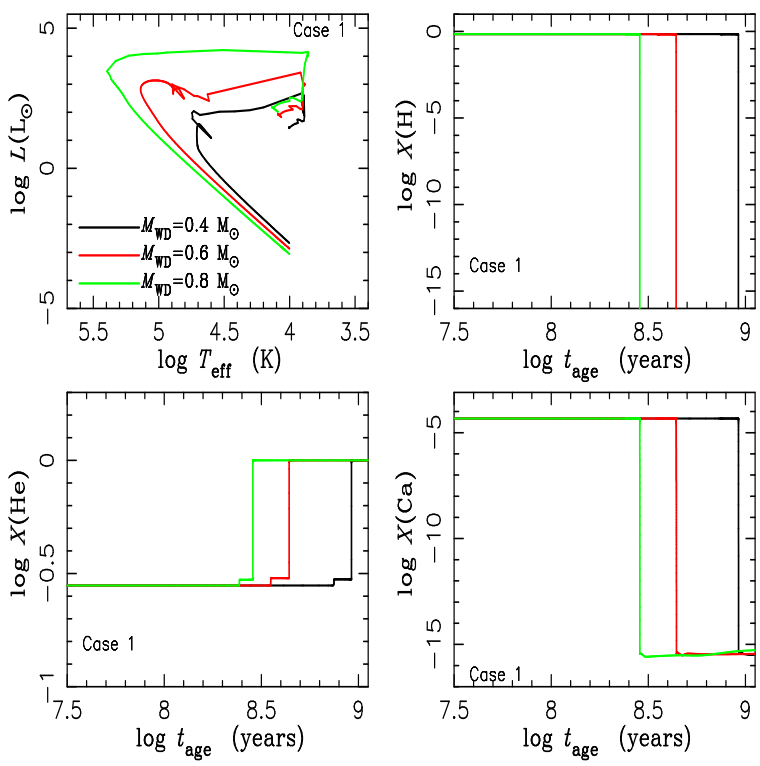

Fig. 2. Similar with Figure 1 but for $0.4,0.6$ and $0.8 \mathrm{M}_{\odot}$ DB WDs produced by MS stars in case 1 with initial masses of $2.5,3.5$ and $5 \mathrm{M}_{\odot}$, which are represented by black, red and green lines, respectively.

ment floats up. For example, $X(\mathrm{Ca})$ on the DB WD's surface has decreased to $10^{-15}$ from initial $10^{-5}$, while a heavy He envelope with mass of about $0.02 \mathrm{M}_{\odot}$ forms around WD surface. Figure 4 gives the profiles of 0.4 and $0.8 \mathrm{M}_{\odot}$ DB WDs for case 1 . Obviously, in our model, He layer mass is affected by WD's mass. It changes from about 0.1 to $0.01 \mathrm{M}_{\odot}$ when $M_{\mathrm{WD}}$ increases from 0.4 to $0.8 \mathrm{M}_{\odot}$.

In Figure 5, we give the change of convective-zone mass $\left(M_{\mathrm{cvz}}\right)$ around WD surface with $T_{\mathrm{eff}}$. For $0.6 \mathrm{M}_{\odot}$ DB WD showed in the left panel of Figure 5, the effects of input parameters on $M_{\mathrm{cvz}}$ can be negligible. The main reasons are as follows:

(i)The mixing length parameter $\alpha_{\mathrm{MLT}}$ has a weak effect on $M_{\mathrm{cvZ}}$ because high density of WDs results a small pressure scale height. For example, it is about $10 \mathrm{~cm}$ for a WD with $T_{\text {eff }}=6$ $\mathrm{kK}$.

(ii) The thermohaline mixing hardly affects the convective zone of DB WDs, while it can significantly affect $M_{\mathrm{cvz}}$ of DA WDs Wachlin et al. 2017; Bauer \& Bildsten 2018). Compared with the latter $\left(10^{-15}-10^{-11} \mathrm{M}_{\odot}\right.$ when $\left.T_{\text {eff }}>10 \mathrm{kK}\right)$ (Koester 2009; Wachlin et al. 2017), $M_{\mathrm{cvz}}$ of DB is much massive, and between about $10^{-9}-10^{-5} \mathrm{M}_{\odot}$. Thick convective zone of DB WDs dilutes the effects of thermohaline mixing, which has been discussed by Bauer \& Bildsten (2019). Simultaneously, Bauer \& Bildsten (2019) mentioned that the mean molecular weight of DB WD is more than two times of DA WD, which dilutes thermohaline mixing effects.

(iii)Metallicity has no effect on $M_{\mathrm{cvz}}$ because the heavy elements rapidly diffuse downward due to the strong gravitational field of WDs.

Compared with $M_{\mathrm{cvz}}$ of $0.6 \mathrm{M}_{\odot}$ DB WD calculated by Benvenuto \& Althaus (1997) and Koester (2009), $M_{\mathrm{cvz}}$ in this work is similar with their results when $T_{\mathrm{eff}}>\sim 14 \mathrm{kK} . M_{\mathrm{cvz}}$ in this work is between that in Benvenuto \& Althaus (1997) and Koester (2009) when $T_{\text {eff }}<\sim 14 \mathrm{kK}$. The right panel of Figure 5 shows $M_{\mathrm{cvz}}$ in the models of 0.4 and $0.8 \mathrm{M}_{\odot}$ DB WDs. Compared with the results of Benvenuto \& Althaus (1997), $M_{\mathrm{cvz}}$ in this work is more massive. The differences mainly result from the following possible aspects:

Firstly, in Benvenuto \& Althaus (1997), the He layer mass of 

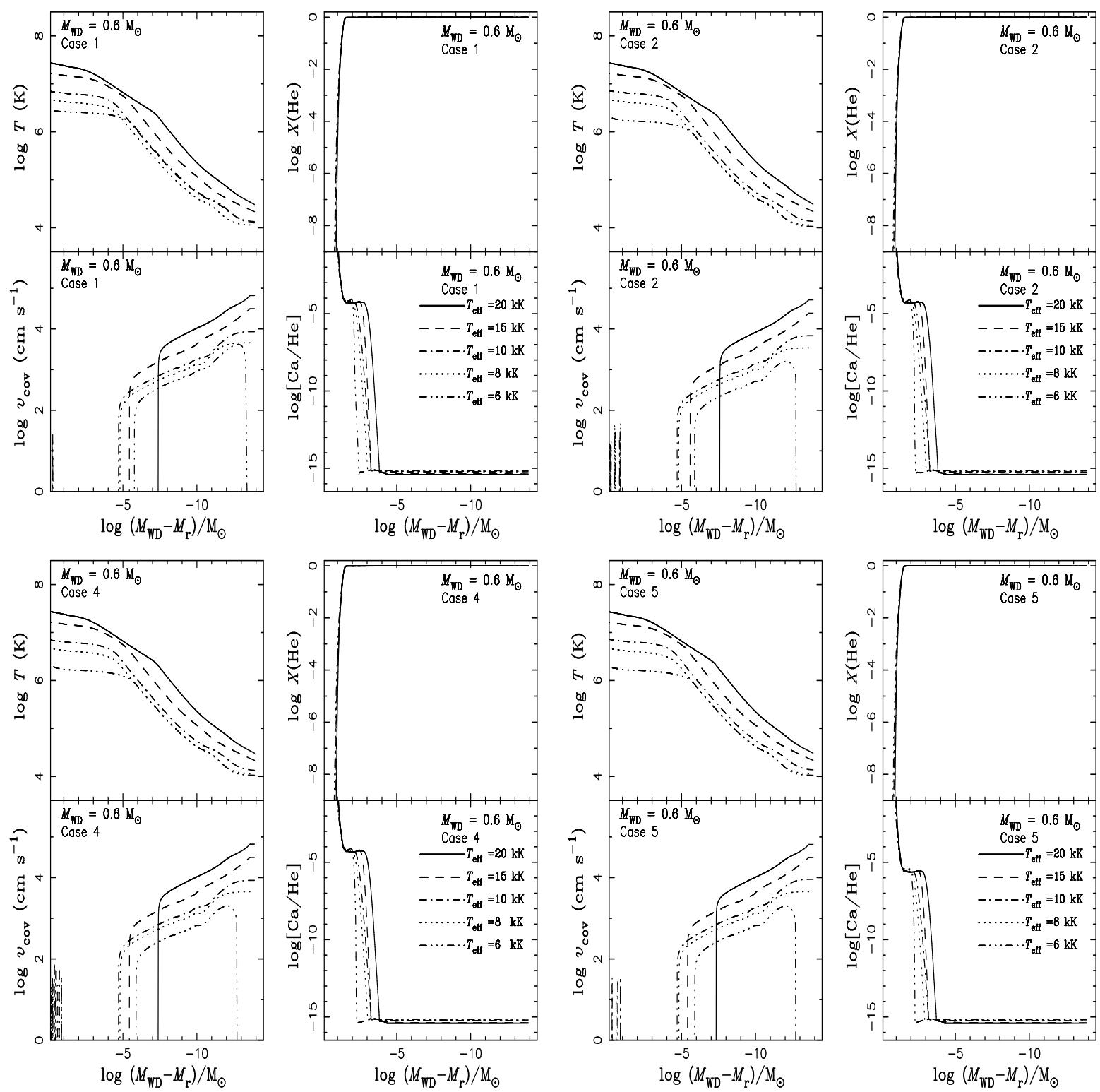

Fig. 3. Structures of $0.6 \mathrm{M}_{\odot}$ DB WDs in different effective temperatures $\left(T_{\text {eff }} \mathrm{s}\right)$ for cases $1,2,4$ and 5 , respectively. The different $T_{\text {eff }} \mathrm{s}$ are given by different lines. $T, v_{\text {cov }}, X(\mathrm{He})$ and $[\mathrm{Ca} / \mathrm{He}]$ represent the temperature, convective velocity, $\mathrm{He}$ abundance and the abundance ratio of $\mathrm{Ca}$ to $\mathrm{He}$, respectively.

DB WDs is between about $10^{-2}$ and $10^{-6} \mathrm{M}_{\odot}$. However, in our work, we consider the gravitational diffusion in DB WDs. The He layer mass is larger than $10^{-2} \mathrm{M}_{\odot}$ and the heavy elements (such as $\mathrm{Ca}$, Fe et al.) sink down. The different chemical profile around WD surface can affect the convective zone.

Secondly, in Benvenuto \& Althaus (1997) and Koester (2009), $M_{\mathrm{cvz}}$ is defined by the thermal time scale. However, $M_{\mathrm{cvz}}$ is defined by Ledoux criterion in our results. As discussed in Koester (2009), $M_{\mathrm{cvz}}$ can differ by orders of magnitude because of different definitions.

\section{Accreting pollution of DB WDs}

The right-bottom panels of Figures 3 and 4 show that $[\mathrm{Ca} / \mathrm{He}]$ on the surface of DB WD has decreases to about $10^{-15}$ due to gravitational settling when $T_{\text {eff }}>20 \mathrm{kK}$. Chayer et al. (1995a) suggested that some element diffusion can be prevented by ra- diative levitation when WD temperature is higher than $20 \mathrm{kK}$ (Chayer et al. 1995b; Chayer 2014). In Figure6, we do a test for $0.6 \mathrm{M}_{\odot}$ DB WD as follows: The gravitational settling is not included when $T_{\text {eff }}$ of cooling WD is higher than $20 \mathrm{kK}$, but it is involved when $T_{\text {eff }}<20 \mathrm{kK}$. We find that $[\mathrm{Ca} / \mathrm{He}]$ rapidly decreases, and can not explain observations. Therefore, the heavy elements observed on the DB WD's surfaces must originate from other sources. The rocky objects tidally disrupted by DB WD are possible source (Farihi 2016).

\subsection{Metal-rich Material Accretion}

In general, the element abundances on the surface of accreting DB WD depend on not only the WD properties, but also massaccretion rates $\left(\dot{M}_{\mathrm{a}}\right)$ and the chemical abundances of accreted material. In order to match the observed properties of G29-38 in Xu et al. (2014), Bauer \& Bildsten (2018) assumed that the mass fractions of $\mathrm{Fe}, \mathrm{O}, \mathrm{Mg}, \mathrm{Si}$ and $\mathrm{Ca}$ were in accreted materials 

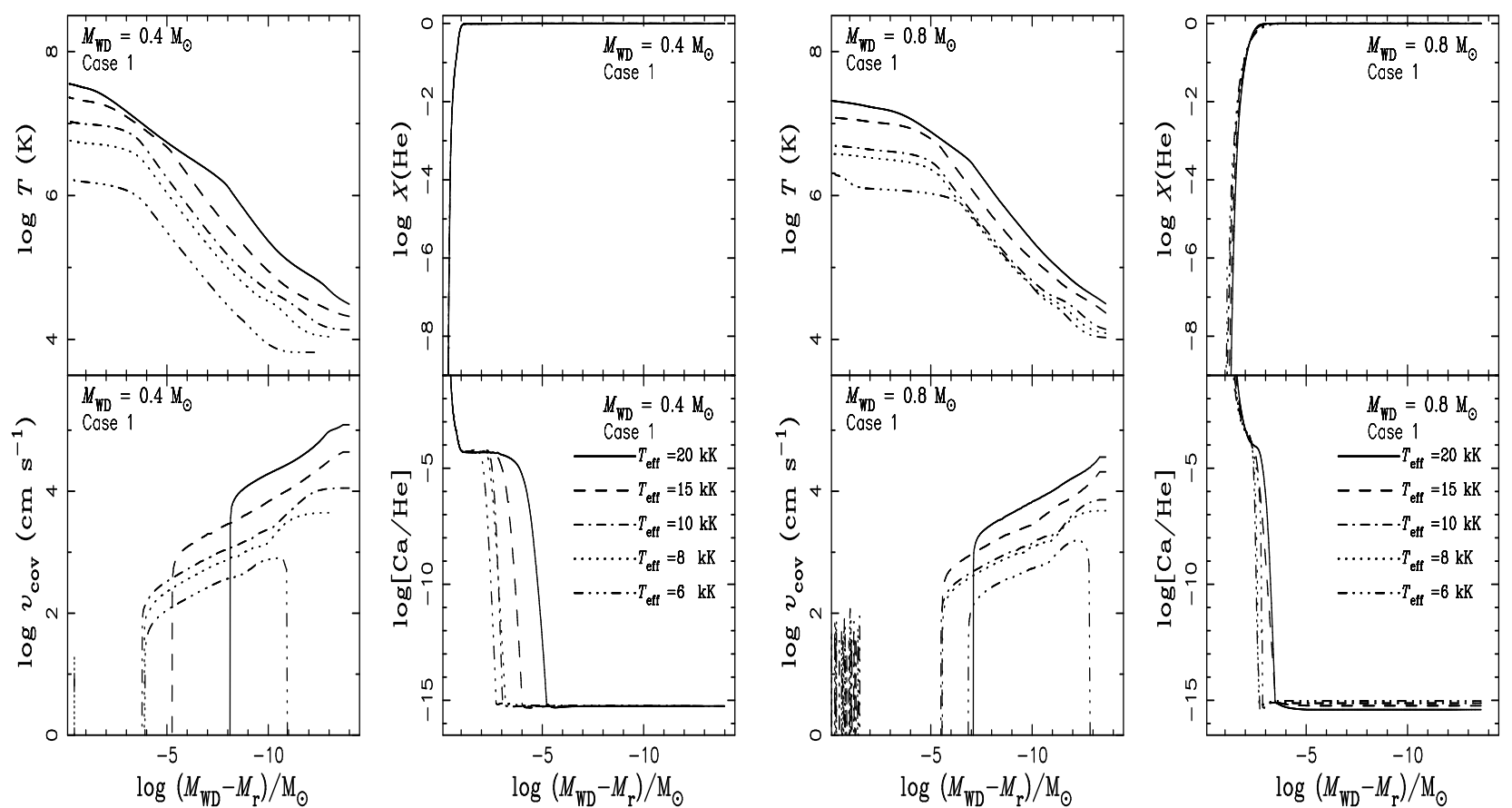

Fig. 4. Similar with Figure 3 but for 0.4 and $0.8 \mathrm{M}_{\odot}$ DB WDs for case 1 .

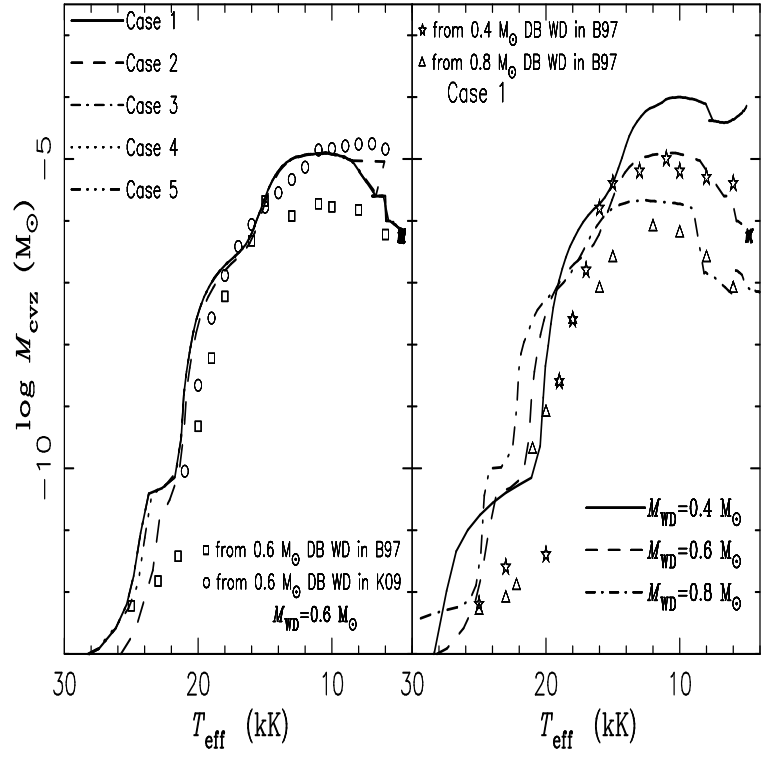

Fig. 5. The mass of convective zone $\left(M_{\text {cvz }}\right)$ vs. the WD's effective temperature $\left(T_{\text {eff }}\right)$. The left panel is for $0.6 \mathrm{M}_{\odot} \mathrm{DB}$ WD in different cases, while right panel is for 0.4, 0.6 and $0.8 \mathrm{M}_{\odot}$ DB WDs in case 1. Theoretical results from Benvenuto \& Althaus (1997) and Koester (2009) are showed by different symbols. B97 and K09 refer to Benvenuto \& Althaus (1997) and Koester (2009), respectively.

\section{$0.307,0.295,0.199,0.153$ and 0.046 , respectively. We adopt the} above mass fractions.

By resolving the Burgers equations, MESA can calculate the chemical diffusion of accreting WD. Figure 7 shows the evolution of $[\mathrm{Ca} / \mathrm{He}]$ on the surface of $0.6 \mathrm{M}_{\odot}$ DB WD with a massaccretion rate of $10^{8} \mathrm{~g} \mathrm{~s}^{-1}$ when $T_{\text {eff }}=20 \mathrm{kK}$. It takes about $10^{4}$ $\mathrm{yr}$ to reach an accretion-diffusion equilibrium for the accreting DB WD. When the accretion stops, Ca element diffuses downward within a diffusive timescale of about $10^{6} \mathrm{yr}$, which is similar with these in Koester (2009). Obviously, input parameters

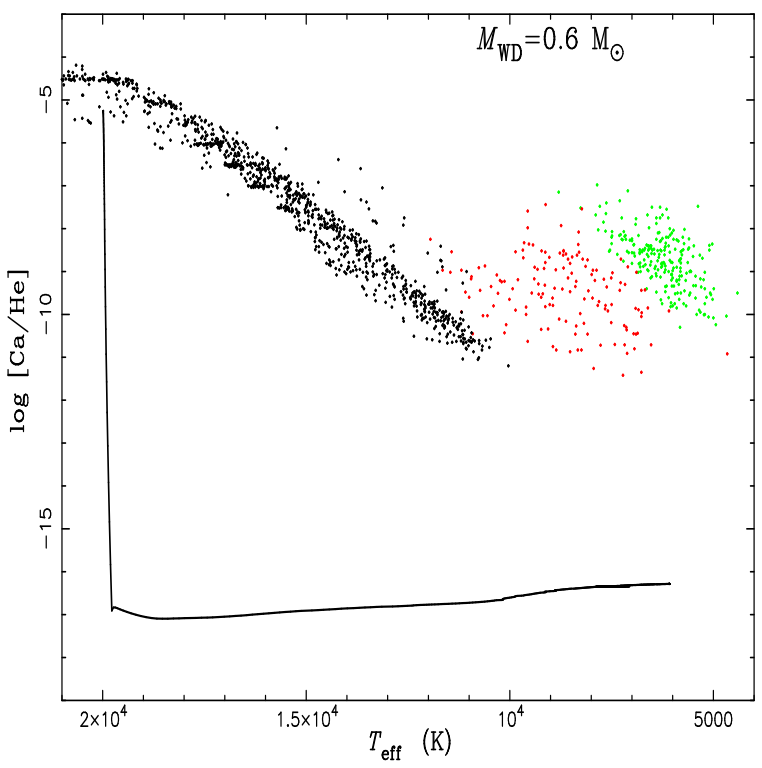

Fig. 6. The evolutions of $\log [\mathrm{Ca} / \mathrm{He}]$ during $0.6 \mathrm{M}_{\odot}$ DB WD cooling, in which the gravitational diffusion is not involved when $T>20 \mathrm{kK}$. Black, red and green dots represent observations from Koester \& Kepler (2015), Dufour et al. (2007) and Hollands et al. (2017), respectively.

( $\alpha_{\mathrm{MLT}}, \alpha_{\text {th }}$ and $Z$ ) have weak effects on the surface $[\mathrm{Ca} / \mathrm{He}]$. The main reasons are similar with these for $M_{\mathrm{cvz}}$.

Figure 8 gives the evolution of $[\mathrm{Ca} / \mathrm{He}]$ on the $0.6 \mathrm{M}_{\odot} \mathrm{DB}$ WD with different $\dot{M}_{\mathrm{a}}$ at different $T_{\text {eff }}$ s. The timescale of reaching accretion-diffusion equilibrium is about $10^{4}$ for all models. The mass-accretion rate and the effective temperature greatly affect the element abundances of accreting DB WD. When $\dot{M}_{\mathrm{a}}$ decreases from $10^{10}$ to $10^{4} \mathrm{~g} \mathrm{~s}^{-1},[\mathrm{Ca} / \mathrm{He}]$ reduces from about $10^{-5}$ to $10^{-10}$. It means that the metal abundance of accreting WD is approximately in proportion to the mass-accretion rate. In fact, Dupuis et al. (1992) and Koester (2009) assumed that the element abundances observed in polluted WDs should be accretion- 


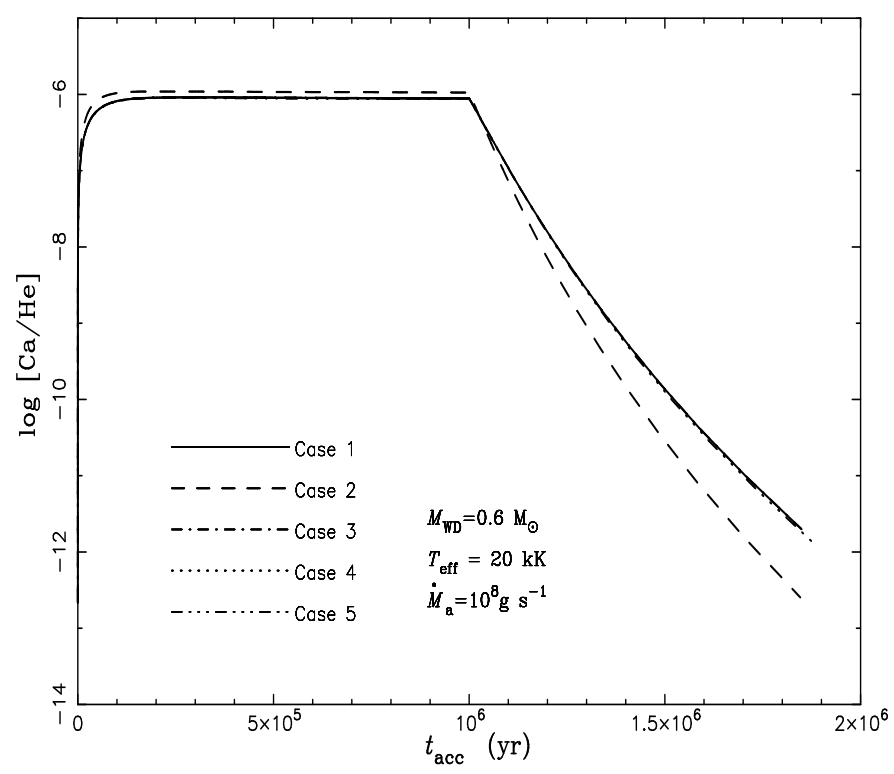

Fig. 7. The evolution of $[\mathrm{Ca} / \mathrm{He}]$ on the surface of $0.6 M_{\odot}$ DB WD with a mass-accretion rate of $10^{8} \mathrm{~g} \mathrm{~s}^{-1}$ when $T_{\text {eff }}=20 \mathrm{kK}$. Accretion ceases after $10^{6}$ years. The different lines represent different cases which showed in the left-bottom zone.

diffusion equilibrium, and they suggested that the mass fraction of the $\mathrm{i}$-th element $\left(X_{\mathrm{cvz}}\right)$ in the convective zone is given by

$M_{\mathrm{cvz}} \frac{\mathrm{d} X_{\mathrm{cvz}, \mathrm{i}}}{\mathrm{d} t}=\dot{M}_{\mathrm{i}}-\frac{X_{\mathrm{cvz}, \mathrm{i}} M_{\mathrm{cvz}}}{\tau_{\mathrm{diff}, \mathrm{i}}}$,

where $X_{\mathrm{cvz}, \mathrm{i}}$ and $\dot{M}_{\mathrm{i}}$ is the i-th element abundance in convective zone and the mass-accretion rate of $i$-th element. Here, $\tau_{\text {diff, } i}$ is the $\mathrm{i}$-th element diffusive timescale, which can be estimated by

$\tau_{\text {diff }, \mathrm{i}}=\frac{M_{\mathrm{cvz}}}{4 \pi R_{\mathrm{B}, \mathrm{cvz}}^{2} \rho_{\mathrm{B}, \mathrm{cvz}} v_{\mathrm{diff}, \mathrm{i}}}$,

where $R_{\mathrm{B}, \mathrm{cvz}}$ and $\rho_{\mathrm{B}, \mathrm{cvz}}$ are the radius and the mass density at the bottom of the convective zone, respectively. Here, $v_{\text {diff, } i}$ is the i-th element velocity of downward sedimentation at bottom of the convective envelope. If $\tau_{\text {diff }}$ is very shorter than WD lifetime, Koester (2009) gave the relation between mass-accretion rate and the element abundance by

$X_{\mathrm{cvz}, \mathrm{i}}=\frac{\dot{M}_{\mathrm{i}}}{M_{\mathrm{cvz}}} \tau_{\mathrm{diff}, \mathrm{i}}$

Obviously, our result is consistent with Eq. (3).

However, the change of $[\mathrm{Ca} / \mathrm{He}]$ with $T_{\text {eff }}$ is complex. When $T_{\text {eff }}$ decreases from 20 to $10 \mathrm{kK},[\mathrm{Ca} / \mathrm{He}]$ reduces by about three orders of magnitude. When it decreases from 10 to $8 \mathrm{kK},[\mathrm{Ca} / \mathrm{He}]$ slightly enhances. When it decreases from 8 to $6 \mathrm{kK},[\mathrm{Ca} / \mathrm{He}]$ reduces by about one order of magnitude again. This change can be explained by the relation of $T_{\mathrm{eff}}$ and $M_{\mathrm{cvz}}$ (See Figure 5).

Figure 9 gives the diffusion downward of $\mathrm{Ca}$ element on the surface of $0.6 \mathrm{M}_{\odot}$ DB WD after a lasting $10^{6} \mathrm{yr}$ accretion at different $T_{\text {eff }}$. The evolution of [Ca/He] with $T_{\text {eff }}$ is similar with that in Figure 8, In fact, Figure 9 indicates the timescale of $\mathrm{Ca}$ element diffusion, that is $\tau_{\text {diff,Ca }}$. Obviously, it deeply depends on $T_{\text {eff }}$. In our model, $\tau_{\text {diff,Ca }}$ increases from about $10^{5}$ to $10^{9} \mathrm{yr}$ when $T_{\text {eff }}$ decreases from 20 to $6 \mathrm{kK}$. However, $\tau_{\text {diff,Ca }}$ in Koester (2009) increases from about $10^{4}$ to $10^{6} \mathrm{yr}$.

Based on Eq. (2), $\tau_{\text {diff,Ca }}$ depends on $M_{\mathrm{cvz}}, R_{\mathrm{B}, \mathrm{cvz}}, \rho_{\mathrm{B}, \mathrm{cvz}}$ and $v_{\text {diff,Ca. }}$. Figure 10 shows the profiles of $X(\mathrm{Ca}), v_{\text {diff,Ca }}$, opacity $(\kappa)$

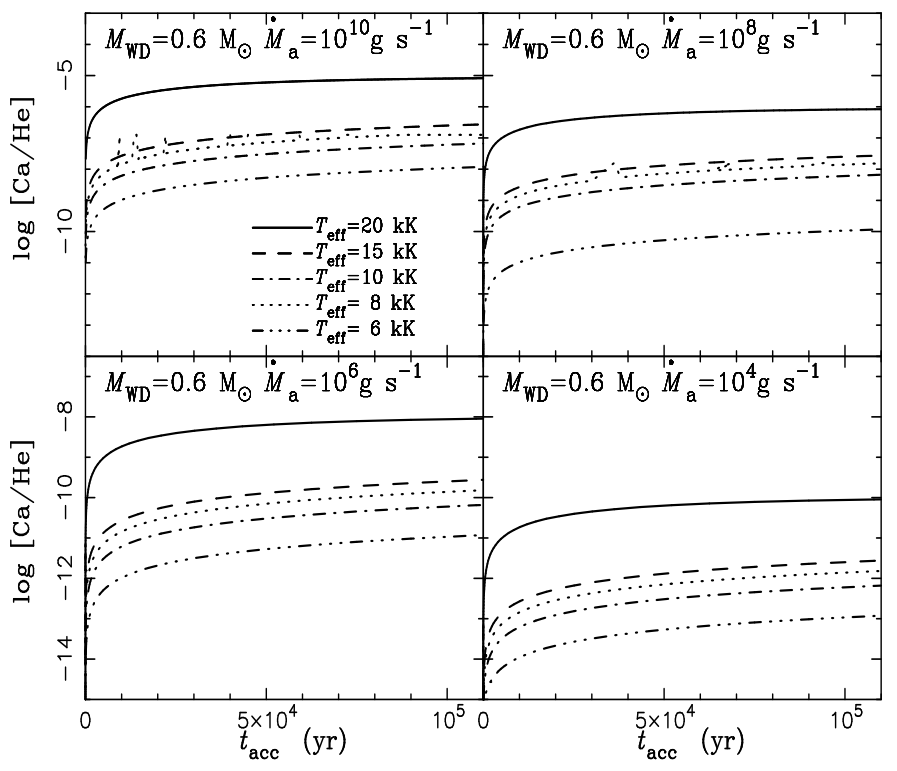

Fig. 8. The evolution of $[\mathrm{Ca} / \mathrm{He}]$ on the surface of $0.6 M_{\odot}$ DB WD with different mass-accretion rates at different $T_{\text {eff }} \mathrm{s}$.

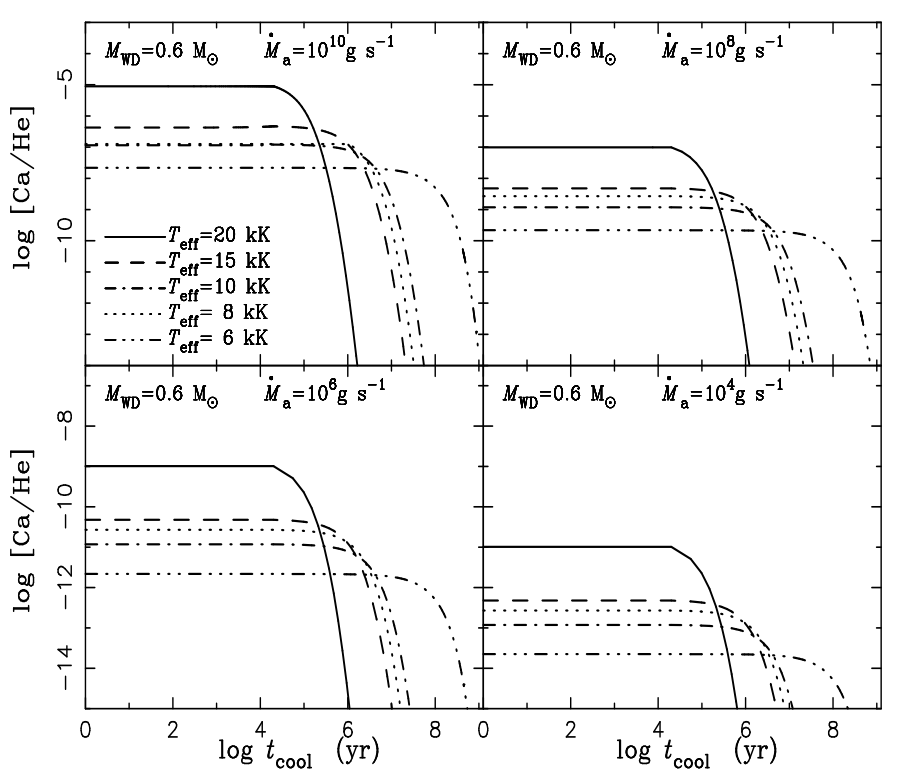

Fig. 9. Similar with Figure 8 but for the evolution of $[\mathrm{Ca} / \mathrm{He}]$ on the surface of $0.6 M_{\odot}$ DB WD which just experiences lasting $10^{6} \mathrm{yr}$ accretion at different $T_{\text {eff. }}$. The mass-accretion rates are showed in the middle-top region of every panel.

and $v_{\text {conv }}$ around the surface of $0.6 \mathrm{M}_{\odot}$ DB WDs with different $\dot{M}_{\mathrm{a}}$ and and $T_{\text {eff. }}$. Obviously, compared model of $10^{8}$ with that of $10^{6} \mathrm{~g} \mathrm{~s}^{-1}$, the mass-accretion rate can affect $X(\mathrm{Ca})$, but does not change the internal structure of the accreting DB WD, including the opacity, the mass density and the radius. The reason is that the matter accreted by DB WD quickly diffuses whole convective zone. DB WD structure mainly depends on cooling duration which is presented by the effective temperature. Due to massive convective zone of DB WDs, compared with He element, abundances of other heavy elements are very low. Therefore, the accreted matter can not affect the internal structure of DB WD, which depends on the cooling duration presented by $T_{\text {eff }}$.

Combining Figures 5, 10 and 11, with DB WD cooling from $T_{\text {eff }}=20 \mathrm{kK}$ to $6 \mathrm{kK}, M_{\mathrm{cvz}}$ increases from about $10^{-8}$ to $10^{-5}$ $\mathrm{M}_{\odot}, \rho_{\mathrm{B}, \mathrm{cvz}}$ also increases from $\sim 1$ to $10^{3} \mathrm{~g} \mathrm{~cm}^{-3}, v_{\mathrm{diff}, \mathrm{Ca}}$ at the 
base of the surface convection zone decreases from about $10^{-6}$ to $10^{-10} \mathrm{~cm} \mathrm{~s}^{-1}$, while $R_{\mathrm{B}, \mathrm{cvz}}$ keeps constant. Therefore, $\tau_{\text {diff,Ca }}$ increases from about $10^{5}$ to $10^{9} \mathrm{yr}$. It means that, compared with $t_{\text {cool }}, \tau_{\text {diff,Ca }}$ can not be neglected when $T_{\text {eff }}<10 \mathrm{kK}$, that is, Eq. (3) is not suitable for cool polluted DB WDs. Of course, one should note, in our model, $v_{\text {diff,Ca }}$ has irregular oscillations when $T_{\text {eff }}<8 \mathrm{kK}$. Koester (2009) did not show $v_{\text {diff,Ca }}$. However, $v_{\text {dif,Ca }}$ may result in great difference of $\tau_{\text {diff,Ca }}$ between the present paper and Koester (2009).

\subsection{Accretion Pollution with Power Law}

As Figure 6 6 shows, the [Ca/He] of about 1500 DB WDs observed by Dufour et al. (2007), Koester \& Kepler (2015) and Hollands et al. (2017) must be explained by accretion pollution. In the panles (a), (b) and (c) of Figure 12, we give the evolutional tracks of $[\mathrm{Ca} / \mathrm{He}]$ with $T_{\text {eff }}$ for DB WDs with masses of $0.4,0.6$ and $0.8 \mathrm{M}_{\odot}$ and constant mass-accretion rates of $10^{10}, 10^{8}$ and $10^{6} \mathrm{~g} \mathrm{~s}^{-1}$, respectively. Obviously, the results hardly explain the observations.

In order to model the metal abundances in GD 362's atmosphere, Jura et al. (2009) provided that $\dot{M}_{\text {a }}$ decreases by power law:

$\dot{M}_{\mathrm{a}}=\frac{M_{\text {disk }}}{t_{\text {disk }}} e^{-t / t_{\text {disk }}}$,

where $M_{\text {disk }}$ is the mass of planet disrupted by WD and $t_{\text {disk }}$ is a characteristic timescale of accretion disk. Jura et al. (2009) found that all of GD 362's distinctive properties can be explained if $M_{\text {disk }}$ is between about $10^{25}$ and $10^{28} \mathrm{~g}$, in which the range of $t_{\text {disk }}$ is between about $2 \times 10^{5}$ and $10^{9} \mathrm{yr}$.

However, based on Figures 8 and panels (a) - (c) of Figure 12 with DB WD cooling, a decreasing mass-accretion rate with power law results in a continued decrease of $[\mathrm{Ca} / \mathrm{He}]$. Therefore, it can not explain the observations. According to our model, the mass-accretion rate should decrease when $T_{\text {eff }}>10 \mathrm{kK}$, but it should increase when $T_{\text {eff }}<10 \mathrm{kK}$. Considering that $T_{\text {eff }}$ of WDs mainly depends on $t_{\text {cool }}$ and can compare with the observations, we assume that $\dot{M}_{\mathrm{a}}$ changes by power law:

$\dot{M}_{\mathrm{a}}=\left\{\begin{array}{lll}10^{14} \times 10^{-4\left(\frac{20 \mathrm{kK}}{T_{\mathrm{eff}}}\right)}, \mathrm{g} \mathrm{s}^{-1} & T_{\mathrm{eff}}>10 \mathrm{kK} \\ 10^{3} \times 10^{3\left(\frac{10 \mathrm{kK}}{T_{\mathrm{eff}}}\right)}, \mathrm{g} \mathrm{s}^{-1} & T_{\mathrm{eff}}<10 \mathrm{kK}\end{array}\right.$

The panel (d) of Figure 12 gives the evolution of $[\mathrm{Ca} / \mathrm{He}]$ with $T_{\text {eff }}$ for DB WDs with different $M_{\mathrm{WD}}$ and an power-law $\dot{M}_{\mathrm{a}}$ described by Eq. (5). Our results are consistent with the observations for DB WDs. The $t_{\text {disk }}$ of an accretion disk composed purely of dust is higher than $10^{9} \mathrm{yr}($ Farihi et al. 2008). Usually, the cooling timescale of DB WD from $20 \mathrm{kK}$ to $10 \mathrm{kK}$ is about $10^{8}-10^{9} \mathrm{yr}$, and it is about $10^{9} \mathrm{yr}$ from $10 \mathrm{kK}$ to $5 \mathrm{kK}$. It means that a DB WD can accrete a disk produced by itself disrupting a planet during the whole cooling phase. The decrease of massaccretion rate when $T_{\text {eff }}>10 \mathrm{kK}$ results from the viscous dissipation of accretion disk(Jura et al. 2009). However, we do not find any model to explain its enhance when $T_{\text {eff }}<10 \mathrm{kK}$. If Eq. (5) basically represents the true trend of the mass-accretion rates, this indicates that the accretion disk produced by WD disrupting a planet may have complex structure.

\section{Conclusions}

In order to explain the evolutionary sequence of heavy element abundances observed among 1500 DB or DZ WDs, we use
MESA to create DB WDs with masses of $0.4,0.6$ and $0.8 \mathrm{M}_{\odot}$ by artificially stripping envelope once. The H-rich envelope is stripped when stars evolve into red giants. We investigate the effects of input parameters ( $\alpha_{\mathrm{MLT}}, \alpha_{\mathrm{th}}$ and $Z$ ) on DB WD structures. Due to the small pressure scale height, thick convective zone or mean molecular weight of DB WDs, these input parameters have weak effect on DB WD structures including interior temperatures, chemical profiles and convective zones. Therefore, they hardly affect the evolution of heavy elements on the surface of DB WDs.

Due to high gravitational fields of DB WDs, the element diffusion in the theoretical model is too fast to explain the observations. Therefore, the heavy elements on the DB WDs' surfaces may originate from the pollution by accreting the planet disrupted by these WDs. They mainly depend on the mass-accretion rates and the effective temperatures of DB WDs. In our model, a constant mass-accretion rate can not explain the evolutionary sequence of Ca element for about 1500 observed DB or DZ WDs. However, it is consistent well with the model in which the massaccretion rate decreases by one power law when $T_{\text {eff }}>10 \mathrm{kK}$ and slightly increases by another power law when $T_{\mathrm{eff}}<10 \mathrm{kK}$. The observed DB WD evolutionary sequence of heavy element abundances originates from WD cooling and the change of massaccretion rate.

Acknowledgements. This work received the generous support of the National Natural Science Foundation of China, project Nos. 11763007, U2031204, and 11863005 .

\section{References}

Bahcall, J. N. \& Loeb, A. 1990, ApJ, 360, 267. doi:10.1086/169116

Bauer, E. B., \& Bildsten, L. 2018, ApJ, 859, L19

Bauer, E. B., \& Bildsten, L. 2019, ApJ, 872, 96

Benvenuto, O. G. \& Althaus, L. G. 1997, MNRAS, 288, 1004. doi:10.1093/mnras/288.4.1004

Berger, L., Koester, D., Napiwotzki, R., et al. 2005, A\&A, 444, 565. doi:10.1051/0004-6361:20053340

Blouin, S., Dufour, P., Thibeault, C., et al. 2019, ApJ, 878, 63

Bohm, K. H. \& Cassinelli, J. 1971, A\&A, 12, 21

Burgers, J. M. 1969, Flow Equations for Composite Gases, New York: Academic Press, 1969

Chambers, K. C., Magnier, E. A., Metcalfe, N., et al. 2016, arXiv:1612.05560

Chayer, P., Vennes, S., Pradhan, A. K., et al. 1995, ApJ, 454, 429

Chayer, P., Fontaine, G., \& Wesemael, F. 1995, ApJS, 99, 189

Chayer, P. 2014, MNRAS, 437, L95

Coutu, S., Dufour, P., Bergeron, P., et al. 2019, ApJ, 885, 74

Deal, M., Deheuvels, S., Vauclair, G., et al. 2013, A\&A, 557, L12

Dufour, P., Bergeron, P., Liebert, J., et al. 2007, ApJ, 663, 1291

Dufour, P., Blouin, S., Coutu, S., et al. 2017, 20th European White Dwarf Workshop, 509, 3

Dupuis, J., Fontaine, G., Pelletier, C., et al. 1992, ApJS, 82, 505

Dupuis, J., Fontaine, G., Pelletier, C., et al. 1993, ApJS, 84, 73

Dupuis, J., Fontaine, G., \& Wesemael, F. 1993, ApJS, 87, 345

Farihi, J. 2016, New A Rev., 71, 9

Farihi, J., Jura, M., \& Zuckerman, B. 2009, ApJ, 694, 805

Farihi, J., Becklin, E. E., \& Zuckerman, B. 2008, ApJ, 681, 1470

Gaia Collaboration, Prusti, T., de Bruijne, J. H. J., et al. 2016, A\&A, 595, A1

Gaia Collaboration, Brown, A. G. A., Vallenari, A., et al. 2018, A\&A, 616, A1

Girven, J., Brinkworth, C. S., Farihi, J., et al. 2012, ApJ, 749, 154

Glebbeek, E., Gaburov, E., de Mink, S. E., et al. 2009, A\&A, 497, 255. doi:10.1051/0004-6361/200810425

Han, Z. 1998, MNRAS, 296, 1019

Han, Z., Tout, C. A., \& Eggleton, P. P. 2000, MNRAS, 319, 215

Han, Z., Podsiadlowski, P., Maxted, P. F. L., et al. 2002, MNRAS, 336, 449

Han, Z., Podsiadlowski, P., Maxted, P. F. L., et al. 2003, MNRAS, 341, 669

Hermes, J. J., Gänsicke, B. T., Kawaler, S. D., et al. 2017, ApJS, 232, 23. doi:10.3847/1538-4365/aa8bb5

Hollands, M. A., Koester, D., Alekseev, V., et al. 2017, MNRAS, 467, 4970

Hollands, M. A., Gänsicke, B. T., \& Koester, D. 2018, MNRAS, 477, 93

Jura, M. 2003, ApJ, 584, L91 

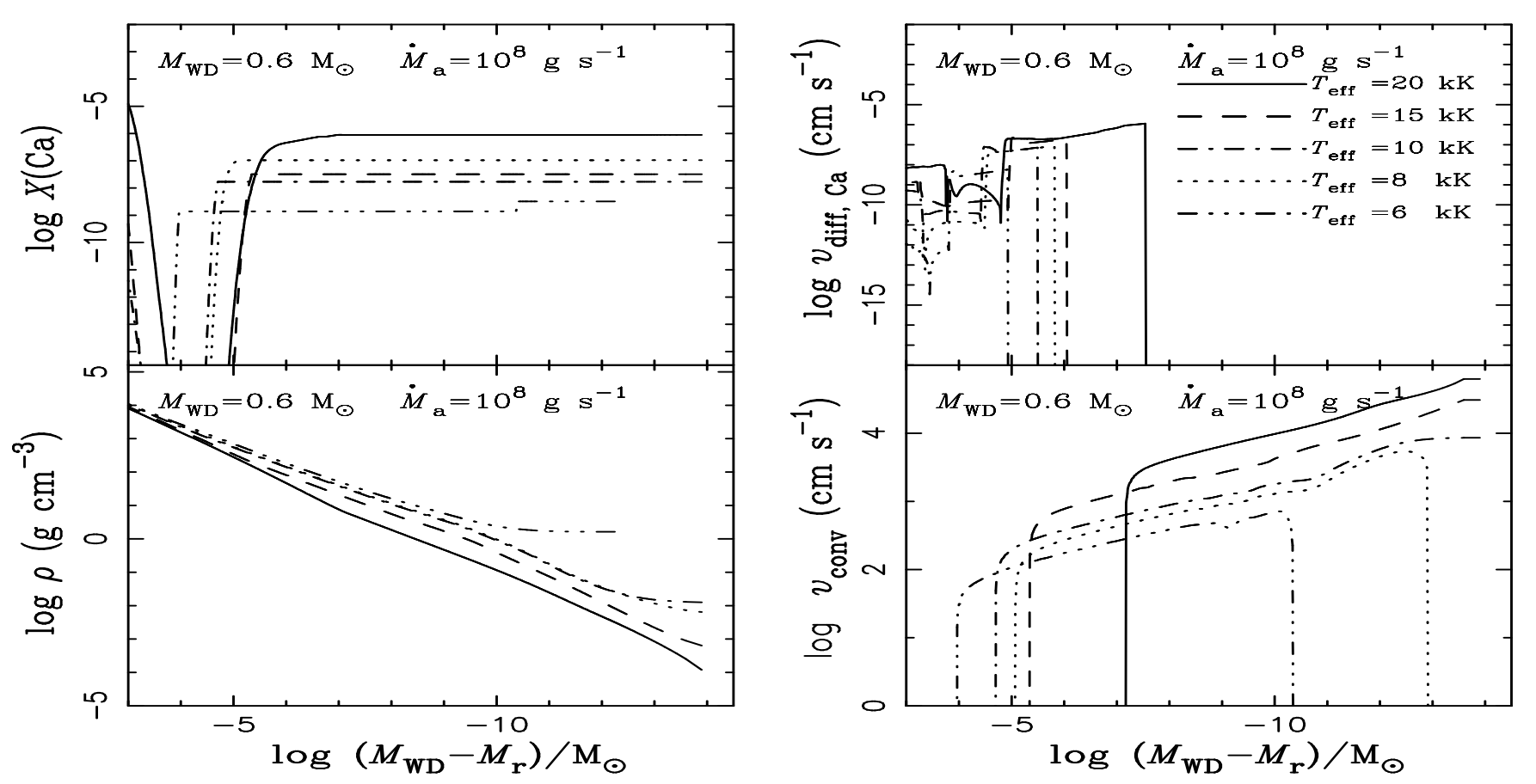

Fig. 10. The profiles of Ca abundance $(X(\mathrm{Ca}))$, opacity $(\kappa)$, Ca diffusive velocity $\left(v_{\text {diff,Ca }}\right)$, convective velocity $\left(v_{\text {conv }}\right)$, mass density $(\rho)$ and radius $(R)$ around the surface of $0.6 M_{\odot}$ DB WDs with a mass-accretion rates of $10^{8} \mathrm{~g} \mathrm{~s}^{-1}$ but different effective temperatures which are represented by different lines.
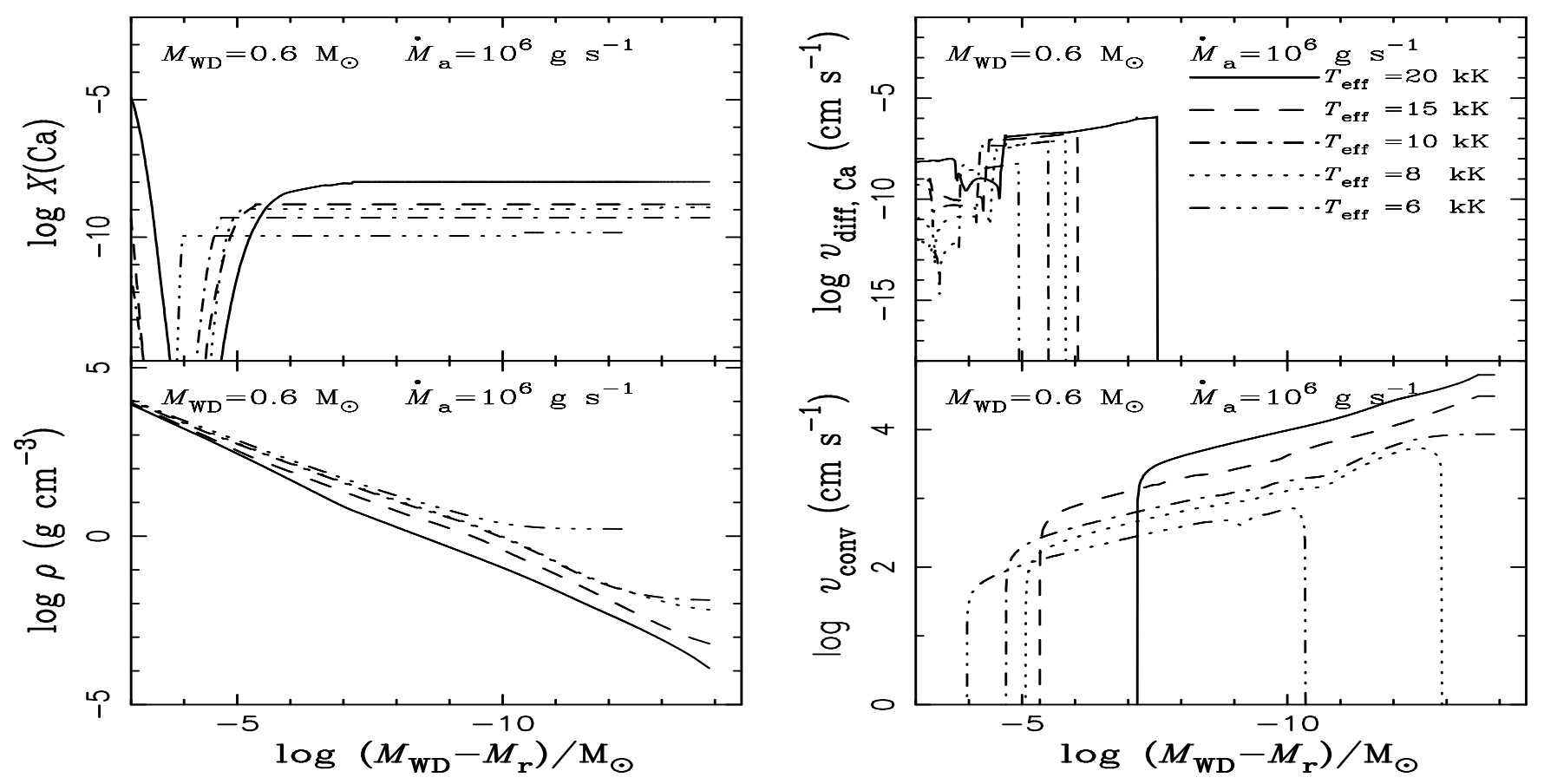

Fig. 11. Similar with Figure 10 but for $\dot{M}=10^{6} \mathrm{~g} \mathrm{~s}^{-1}$.

Jura, M. \& Young, E. D. 2014, Annual Review of Earth and Planetary Sciences, 42,45

Jura, M., Muno, M. P., Farihi, J., et al. 2009, ApJ, 699, 1473

Kawaler, S. D. 2015, 19th European Workshop on White Dwarfs, 493, 65

Kippenhahn, R., Ruschenplatt, G., \& Thomas, H.-C. 1980, A\&A, 91, 175

Koester, D. \& Wilken, D. 2006, A\&A, 453, 1051

Koester, D. 2009, A\&A, 498, 517

Koester, D., Gänsicke, B. T., \& Farihi, J. 2014, A\&A, 566, A34

Koester, D. \& Kepler, S. O. 2015, A\&A, 583, A86

Koester, D., Kepler, S. O., \& Irwin, A. W. 2020, A\&A, 635, A103

Lü, G., Zhu, C., Wang, Z., et al. 2017, ApJ, 847, 62

Lü, G., Zhu, C., Wang, Z., et al. 2020, ApJ, 890, 69

Nieuwenhuijzen, H. \& de Jager, C. 1990, A\&A, 231, 134

Nugis, T. \& Lamers, H. J. G. L. M. 2000, A\&A, 360, 227 Paxton, B., Bildsten, L., Dotter, A., et al. 2011, ApJS, 192, 3

Paxton, B., Cantiello, M., Arras, P., et al. 2013, ApJS, 208, 4

Paxton, B., Marchant, P., Schwab, J., et al. 2015, ApJS, 220, 15

Paxton, B., Schwab, J., Bauer, E. B., et al. 2018, ApJS, 234, 34

Paxton, B., Smolec, R., Schwab, J., et al. 2019, ApJS, 243, 10

Paquette, C., Pelletier, C., Fontaine, G., et al. 1986, ApJS, 61, 177

Reimers, D. 1975, Memoires of the Societe Royale des Sciences de Liege, 8, 369

Schatzman, E. 1945, Annales d'Astrophysique, 8, 143

Shapiro, S. L. \& Teukolsky, S. A. 1983, A Wiley-Interscience Publication, New York: Wiley, 1983

Stanton, L. G. \& Murillo, M. S. 2016, Phys. Rev. E, 93, 043203

Tassoul, M., Fontaine, G., \& Winget, D. E. 1990, ApJS, 72, 335. doi: $10.1086 / 191420$

Article number, page 8 of 9 

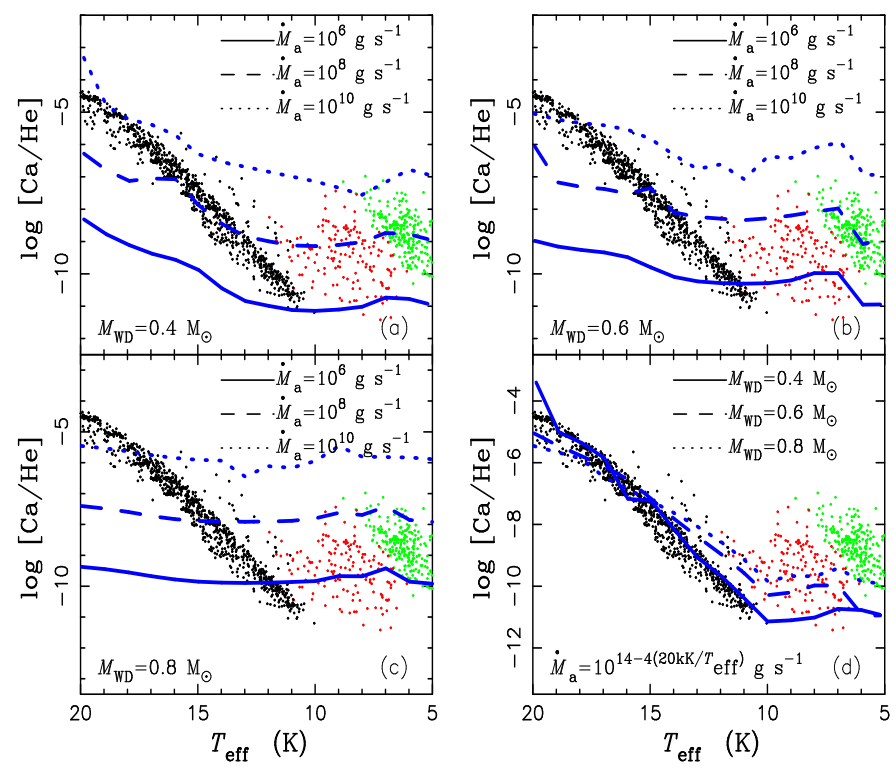

Fig. 12. Effective temperature vs. $\log [\mathrm{Ca} / \mathrm{He}]$ for $\mathrm{DB}$ WDs. Panels (a), (b), and (c) represent models with different mass DB WDs and a constant accretion rate $\left(10^{6}, 10^{8}\right.$ and $\left.10^{10} \mathrm{~g} \mathrm{~s}^{-1}\right)$, respectively. Panels (d) is for the mass-accretion rate given by Eq. (5). Black, red and green dots represent observations from Koester \& Kepler (2015), Dufour et al. (2007) and Hollands et al. (2017), respectively.

Thoul, A. A., Bahcall, J. N., \& Loeb, A. 1994, ApJ, 421, 828

Vanderburg, A., Johnson, J. A., Rappaport, S., et al. 2015, Nature, 526, 546

Vink, J. S., de Koter, A., \& Lamers, H. J. G. L. M. 2001, A\&A, 369, 574 doi:10.1051/0004-6361:20010127

Voss, B., Koester, D., Napiwotzki, R., et al. 2007, A\&A, 470, 1079

Wachlin, F. C., Vauclair, G., Vauclair, S., et al. 2017, A\&A, 601, A13

Xu, S., Jura, M., Koester, D., et al. 2014, ApJ, 783, 79

Yu, J., Li, Z., Zhu, C., et al. 2019, ApJ, 885, 20

Zhu, C., Liu, H., Lü, G., et al. 2019, MNRAS, 488, 525

Zuckerman, B., Koester, D., Reid, I. N., et al. 2003, ApJ, 596, 477

Zuckerman, B., Koester, D., Melis, C., et al. 2007, ApJ, 671, 872 